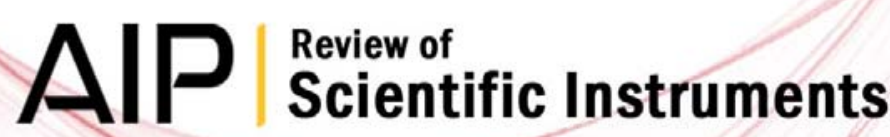

\section{Brandaris 128 ultra-high-speed imaging facility: 10 years of operation, updates, and enhanced features}

Erik C. Gelderblom, Hendrik J. Vos, Frits Mastik, Telli Faez, Ying Luan et al.

Citation: Rev. Sci. Instrum. 83, 103706 (2012); doi: 10.1063/1.4758783

View online: http://dx.doi.org/10.1063/1.4758783

View Table of Contents: http://rsi.aip.org/resource/1/RSINAK/v83/i10

Published by the American Institute of Physics.

\section{Related Articles}

A fast real time measurement system to track in and out of plane optical retardation/ birefringence, true stress, and true strain during biaxial stretching of polymer films

Rev. Sci. Instrum. 83, 123901 (2012)

Momentum spectrometer for electron-electron coincidence studies on superconductors

Rev. Sci. Instrum. 83, 103905 (2012)

Thermal imaging diagnostics of high-current electron beams

Rev. Sci. Instrum. 83, 103301 (2012)

A holistic approach performance analysis of substrate-free focal plane array J. Appl. Phys. 112, 074502 (2012)

Noise characteristics of short wavelength infrared InGaAs linear focal plane arrays J. Appl. Phys. 112, 064509 (2012)

\section{Additional information on Rev. Sci. Instrum.}

Journal Homepage: http://rsi.aip.org

Journal Information: http://rsi.aip.org/about/about_the_journal

Top downloads: http://rsi.aip.org/features/most_downloaded

Information for Authors: http://rsi.aip.org/authors

\section{ADVERTISEMENT}

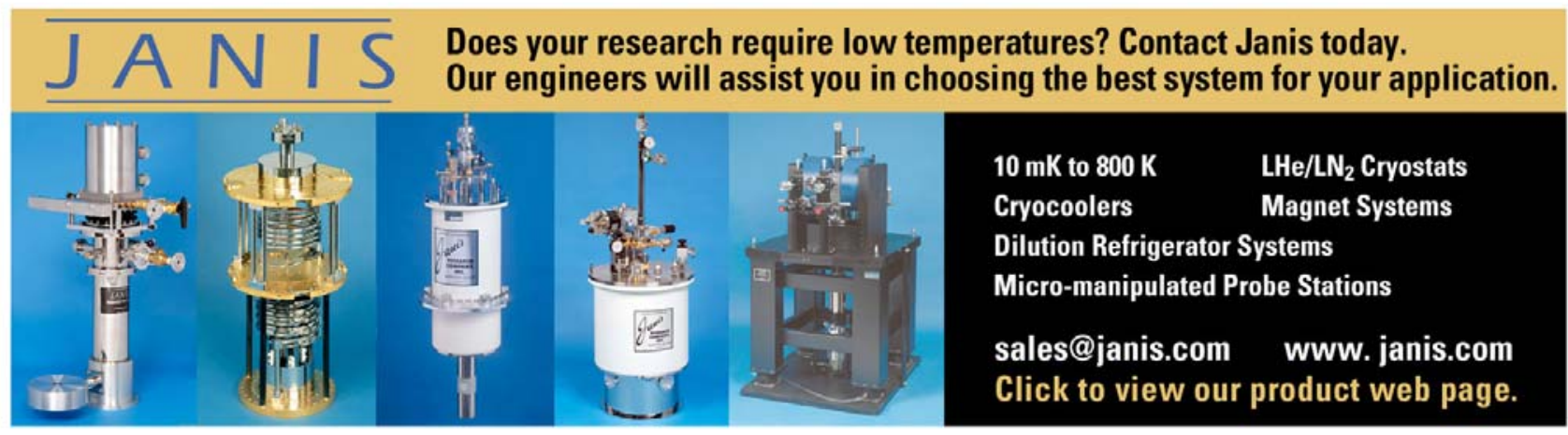




\title{
Brandaris 128 ultra-high-speed imaging facility: 10 years of operation, updates, and enhanced features
}

\author{
Erik C. Gelderblom, ${ }^{1}$ Hendrik J. Vos, ${ }^{2}$ Frits Mastik, ${ }^{2}$ Telli Faez, ${ }^{2}$ Ying Luan, ${ }^{2}$ \\ Tom J. A. Kokhuis, ${ }^{2}$ Antonius F. W. van der Steen, ${ }^{2}$ Detlef Lohse, ${ }^{1}$ Nico de Jong, ${ }^{1,2}$ \\ and Michel Versluis ${ }^{1}$ \\ ${ }^{1}$ Physics of Fluids Group, MIRA Institute for Biomedical Technology and Technical Medicine, \\ University of Twente, Enschede, 7500 AE, The Netherlands \\ ${ }^{2}$ Biomedical Engineering, Erasmus MC, Rotterdam, 3000 CA, The Netherlands
}

(Received 17 June 2012; accepted 27 September 2012; published online 25 October 2012)

\begin{abstract}
The Brandaris 128 ultra-high-speed imaging facility has been updated over the last 10 years through modifications made to the camera's hardware and software. At its introduction the camera was able to record 6 sequences of 128 images $(500 \times 292$ pixels $)$ at a maximum frame rate of $25 \mathrm{Mfps}$. The segmented mode of the camera was revised to allow for subdivision of the 128 image sensors into arbitrary segments (1-128) with an inter-segment time of $17 \mu \mathrm{s}$. Furthermore, a region of interest can be selected to increase the number of recordings within a single run of the camera from 6 up to 125 . By extending the imaging system with a laser-induced fluorescence setup, time-resolved ultra-highspeed fluorescence imaging of microscopic objects has been enabled. Minor updates to the system are also reported here. (C) 2012 American Institute of Physics. [http://dx.doi.org/10.1063/1.4758783]
\end{abstract}

\section{INTRODUCTION}

Ten years after its introduction, the Brandaris 128 ultrahigh-speed imaging facility ${ }^{1}$ is still the fastest digital highspeed camera in the world to record more than 100 consecutive frames. Employing a rotating mirror, mounted on a helium-driven turbine, a maximum frame rate of $25 \times 10^{6}$ frames per second (Mfps) is achieved. Other cameras, equipped with multiple synchronized sensors, are able to reach higher frame rates up to $200 \mathrm{Mfps}$, using specialized image intensifiers that can be gated down to $5 \mathrm{~ns}$. However, the number of frames within a single recording is limited to 16 in those cameras.

The Brandaris 128, shown in Figure 1, is based on the camera frame of the Cordin 119 (Cordin Scientific Imaging, Salt Lake City, UT). The rotating mirror camera design allows for a high frame rate and high number of frames, while the CCD sensors offer superior recording sensitivity and flexibility. Various lenses can be mounted to suit the experimental limitations, ranging from microscope objectives to single-lens reflex camera objectives. The primary image of an object is projected onto the rotating mirror prism by two relay lenses. The three-sided mirror is mounted onto a helium-driven turbine to redirect the image through the lens bank onto the CCD sensors.

The optical configuration is schematically depicted in Figure 2(a). The Miller principle for high-speed cinematography ${ }^{5}$ was applied to obtain stationary images while the mirror sweeps the light beam across the 128 lens pairs in the lens bank, which refocus the image onto 128 highly sensitive, un-intensified CCD image sensors (Sony ICX-055AL, $500 \times 292$ pixels). The field lens and aperture are used to match the numerical aperture (NA) of the light beam to the NA of the lens pairs, thereby setting the exposure time per channel. As the mirror rotates, the image formed on a single detector changes slightly. The resulting blur, called image drag, is minimized by projecting the primary image as close as possible onto the apparent axis of rotation of the mirror. ${ }^{6}$ A maximum image drag of $7.5 \mu \mathrm{m}$ is produced by the optical configuration and $50 \%$ of the channels suffer an image drag of less than $4 \mu \mathrm{m}$, which is smaller than the pixel height of the CCDs $(12 \mu \mathrm{m})$ in the lateral direction.

A mass flow controller (MFC) (F-206AI, Bronkhorst, Ruurlo, The Netherlands) was installed to regulate the flow of helium to the turbine. Before an experiment is performed the camera case is pre-filled with helium to reduce the viscous drag on the turbine rotation. The maximum turbine speed of $20000 \mathrm{rps}$ is achieved at a flow rate of $2500 \mathrm{l} / \mathrm{min}$ and results in a frame rate of $25 \mathrm{Mfps}$.

A custom designed CCD controller card $\left(\mathrm{C}^{3}\right)$ coordinates the image capture process for every four CCD detectors. Several microseconds before exposure of the first channel, all CCDs are flushed to drain the accumulated charge in the photosensitive cells. After exposure of the last channel a charge transfer shifts the photoelectrons into the transport channels of the CCDs, preventing multiple exposure of the CCDs. After the charge transfer all pixels are read out for analogueto-digital conversion and the image data are stored in the onboard RAM of the CCDs. Flush and transfer only take several microseconds to perform, whereas readout takes $80 \mathrm{~ms}$ to finish. When the readout is completed another recording can be made. The onboard RAM of the CCDs limits the number of recordings within one experiment to 6 , leading to a total number of 768 full frames. After the final readout, all image data are transferred via USB-hubs to a desktop computer for data storage and analysis. Since this takes only several seconds, many consecutive experiments can be performed in a short time frame.

A single experiment performed with the Brandaris 128 in default mode consists of 6 recordings, using all 128 frames for each recording, as depicted in Figure 2(b). To increase the 


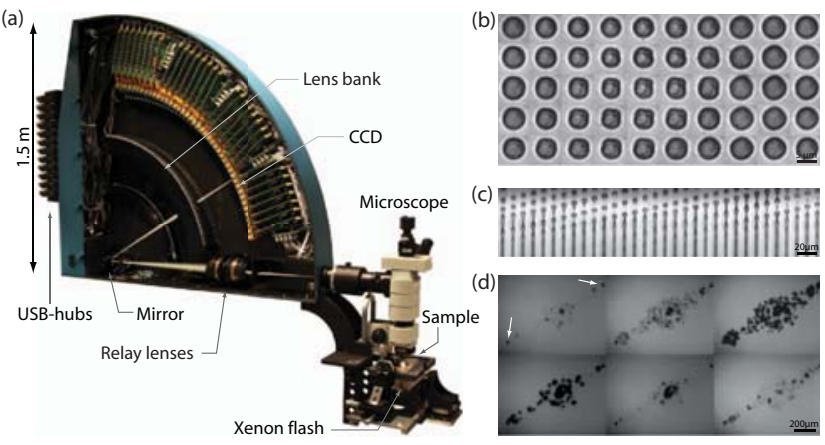

FIG. 1. (a) The Brandaris 128 ultra-high-speed imaging facility. (b) Ultrasound contrast agent showing buckling behavior recorded at $14.5 \mathrm{Mfps}^{2}$ (c) Breakup of a $1.25 \mu \mathrm{m}$ radius liquid jet into microdroplets, visualized with an interframe time of $73 \mathrm{~ns}^{3}$ (d) Cleaning of a silicon wafer by the growth and collapse of a cloud of cavitation bubbles located in between two micropits (white arrows), recorded at 5.4 Mfps. ${ }^{4}$

repetition rate of subsequent recordings a segmented mode was devised, as shown in Figure 2(c). The total number of 128 frames can be divided into 2 segments of 64 frames or 4 segments of 32 frames. After exposure of the first segment, only the charge of the first segment is transferred into the transport channels of the corresponding CCDs. During a subsequent mirror period, all CCDs are flushed again and after exposure the second segment undergoes charge transfer. The data in the transport channels of the first segment are unaffected by the additional exposures, flushes, and charge transfers. After all charge transfer is completed for the last segment, readout can be initiated for all channels. In theory, an intersegment time as short as the mirror period divided by the number of mirror faces can be achieved, resulting in $17 \mu \mathrm{s}$ for a three-sided mirror.

The Brandaris 128 has been dedicated predominantly to the study of medical ultrasound contrast agents. These gas-filled, micrometer-sized, coated bubbles are very efficient scatterers of ultrasound and are used in organ perfusion imaging. The understanding of the interaction of the bubbles and ultrasound allows us to improve their performance. However,
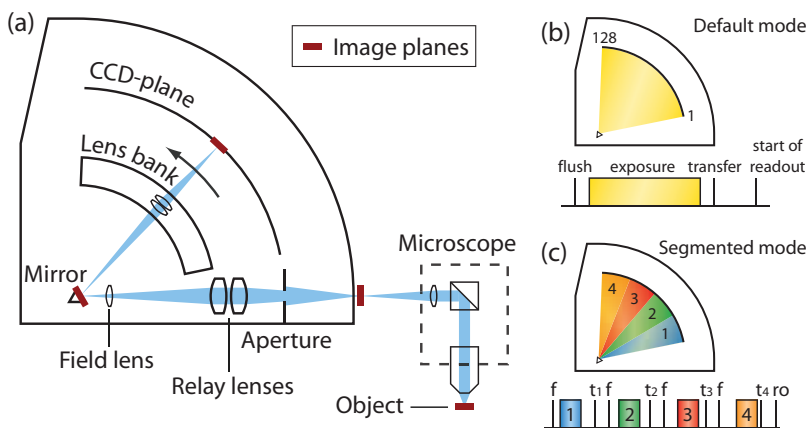

FIG. 2. (a) Schematic representation of the optical configuration of a rotating mirror framing camera. (b) Exposure diagram for a recording in default mode. (c) Exposure diagram for a recording in segmented mode ( $\mathrm{f}=$ flush, $\mathrm{t} \sharp=$ transfer of segment $\sharp$, ro $=$ start of readout).

to achieve this, a detailed insight of the microbubble dynamics is crucial. Since the applied ultrasound frequencies and corresponding microbubble oscillations are in the order of 1-5 MHz, ultra-high-speed imaging at a frame rate exceeding $10 \mathrm{Mfps}$ is required.

The microbubbles are resonators to the applied sound field, hence their response is a function of both pressure and frequency. By systematically varying the ultrasound parameters and recording the microbubbles oscillations, the response of single phospholipid-coated microbubbles is now well understood. A major contribution was made by Van der Meer et $a l^{7}$ in 2007 when the camera was operated in segmented mode. This allowed for the recording of 12 24 sequences in a single experiment to fully capture a bubble's resonance curve within a single run of the camera by changing the insonation frequency in each segment, as shown in Figure 3. This method was termed microbubble spectroscopy, since a single experiment revealed the characteristics of a single microbubble for a scan of a chosen parameter. Other studies visualized for the first time phenomena such as shell buckling, " "compression-only" behavior," "thresholding" behavior, ${ }^{10}$ nonspherical oscillations, ${ }^{11}$ and subharmonic oscillations. $^{12}$
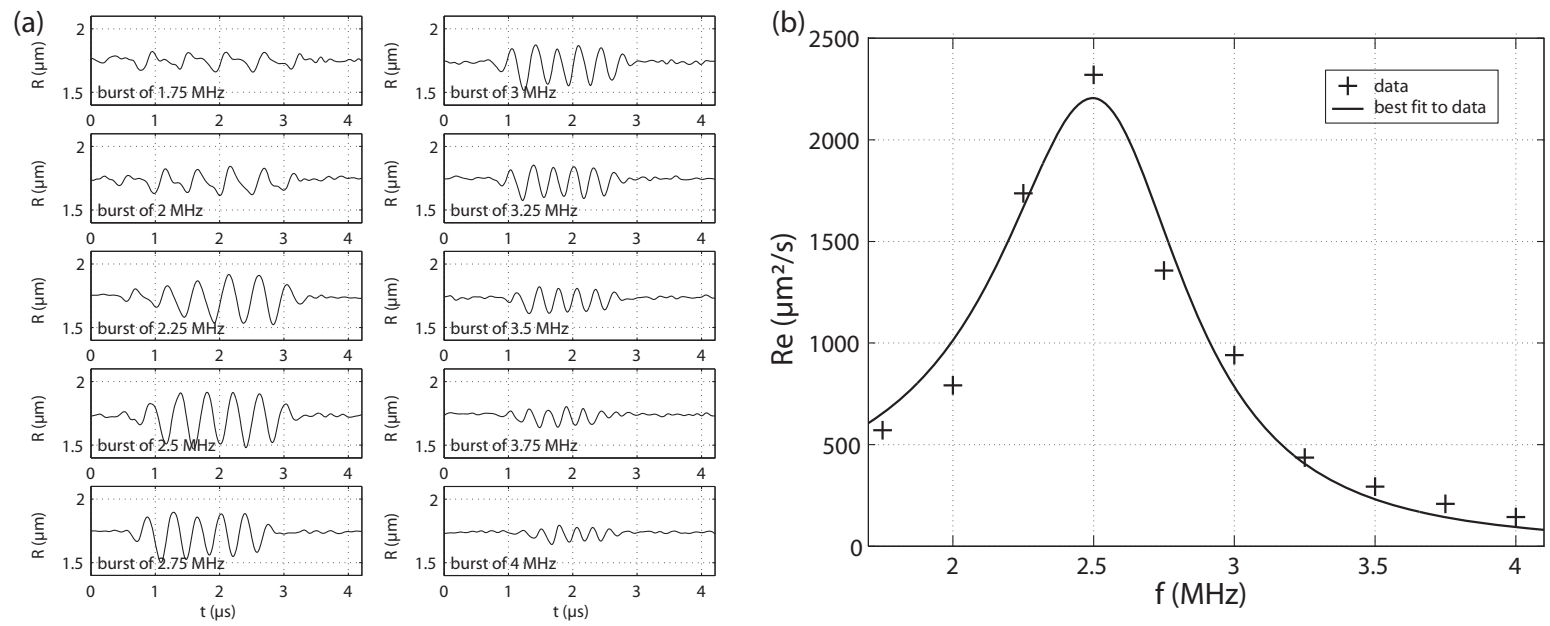

FIG. 3. Microbubble spectroscopy. ${ }^{7}$ (a) Radius-time curves of a microbubble for different excitation frequencies, recorded in a single run of the Brandaris 128. (b) Resonance curve obtained from the radius-time curves of a single run. 
Enhanced functionality was achieved by combining the camera with optical tweezers to perform 3D micromanipulation of microbubbles. ${ }^{13}$ This enabled the comparison of bubble oscillations near a wall and at a distance from the wall within a single experiment, by applying a LaguerreGaussian laser beam to control the vertical position of a microbubble. In the same way, the influence of neighboring bubbles was studied by controlled variation of the distance between two bubbles.

New insight into the physical mechanisms of ultrasoundtriggered local drug delivery was found by studying the release and uptake of drugs by cultured cells. Recordings of a hard-shelled, oil-filled agent showed the disruption of the polymer wall and subsequent release of the contents. ${ }^{14}$ Van Wamel et al. studied the interaction of a microbubble and an endothelial cell. ${ }^{15}$ The high-speed recordings revealed the mechanical forces that a bubble can exert on the neighboring cell membrane, by visualizing the cell deformation following microbubble oscillations. In combination with simultaneous fluorescence recordings, an enhanced permeability of the cell membrane was confirmed and was attributed to acoustic streaming.

The Brandaris 128 has also been used to study other fast phenomena that occur on a nanoseconds time scale, such as droplet formation in piezoinkjet printing ${ }^{16}$ and pulmonary drug delivery, ${ }^{3}$ and bubble-induced cavitation for the cleaning of silicon wafer surfaces. ${ }^{17}$ Figures $1(b)-1(d)$ show several examples of observed phenomena.

\section{A. System limitations}

Despite the numerous novel scientific results obtained with the Brandaris 128, the camera still has its limitations, the first being the recording speed. An increase in frame rate would open up a range of possible applications. Even a "minor" improvement of a factor 2 (50 Mfps) would enable the study of microbubbles excited by ultrasound frequencies in the order of $20 \mathrm{MHz}$. These frequencies are being used for high-resolution intravascular ultrasound imaging. At this point, increasing the frame rate can only be achieved through major alterations to the design of the camera, which, in terms of costs, would be in the same order of a full redesign of the system.

Second, the original timing controller of the Brandaris 128 that generated the triggers for the ultrasound, the illumination and the flush and transfer of the CCDs, was based on manual switches and timer/counter equipment. It required at least two people to operate and the response time was approximately $2 \mathrm{~s}$. To ensure accurate timing, the turbine had to be run for an extended period of time until the speed had stabilized.

Each channel has the capacity to store 6 images in its onboard RAM. It enables the user to perform 6 recordings in less than a second, which is an advantage over other highspeed cameras that can typically only record 1 to sometimes 2 images per sensor. Nevertheless, even 6 recordings are insufficient to capture the dynamics of a microbubble for a small set of three different pressures at three different insonation frequencies. Full characterization then requires multiple experiments taking several minutes to complete in which the properties of the microbubbles may change significantly. This problem can be addressed by using 24 recordings in the segmented mode, however, this limits the number of frames per recording to 32 .

Aside from the reduced number of frames per recording, when it was first introduced the segmented mode was also limited in its inter-segment time. Since no actual readout of the sensors is required until all segments are illuminated, the theoretical time between subsequent segments is the revolution period of the mirror divided by the number of mirror faces. However, these specifications were not met, resulting in a time between segments equal to the time between full sequence recordings, i.e., $80 \mathrm{~ms}$. Furthermore, the number of segments was limited to four.

Finally, for imaging at a micrometer length scale, the typical technique used is bright field microscopy using back illumination, since it has the highest yield in light intensity. At an exposure time of $40 \mathrm{~ns}$ and magnifications up to 200 times, every photon counts. By using a special xenon strobe, bright field sequences can be recorded up to the limiting frame rate of the camera. At frame rates above $8 \mathrm{Mfps}$, the ignition peak of the flash is used, whereas at lower frame rates the longer and more stable discharge pulse of the flash offers sufficient illumination intensity (pulse shape shown schematically in Figure 10). Although the ignition peak is not constant in time, no other light source offers comparable illumination levels for a duration of 5-16 $\mu \mathrm{s} .{ }^{18}$ The varying intensity levels for each individual frame never proved a problem for data analysis. When the setup does not allow for bright field imaging and reflected instead of transmitted light is required, for instance, using dark field illumination, the signal-to-noise ratio (SNR) of the images is decreased significantly. The photon flux reflected by the sample is a factor of 10 lower as compared to back illumination. Using a standard fluorescence microscope, an exposure time in the order of tens of milliseconds is required to visualize a fluorescently labeled microbubble. When fluorescence imaging is required for particle tracking or to visualize drug release or uptake from a microbubble at a (sub)microsecond time scale, coupling the camera to a fluorescence microscope setup simply does not yield enough photons. The reduced exposure time requires an increase of the photon flux of five orders of magnitude to achieve a satisfactory SNR.

\section{B. Enhanced features}

\section{Timing}

To overcome several of the aforementioned limitations, adaptations have been made to both the hardware and software of the camera in the last decade. First, a dedicated timing controller was developed for generating "real-time" trigger signals for illumination, ultrasound, and the CCDs. By constantly monitoring the mirror speed and adapting the pretrigger delays for various devices, a timing accuracy of less than $40 \mathrm{~ns}$ was achieved, equal to the interframe time at the maximum frame rate. As variations in the mirror speed are 
now accounted for continuously, the user does not have to wait for the turbine to run at a stable speed before starting the actual experiment, thereby reducing the time required to reach the desired frame rate and limiting the wear of the turbine. Furthermore, the system was designed to be operated by autonomous triggering at a preset frame rate.

\section{Storage}

An improvement was made with the goal to increase the number of recordings within a single run, which is limited by the storage capacity of the CCDs. In many experiments, only a single bubble or an event that is small compared to the full image frame is of interest. Nevertheless a full frame will be stored in the onboard RAM. By letting the user select a region of interest (ROI) on the CCDs, the image size can be decreased, overcoming the memory space limit of 6 images per channel. This drastically increases the number of recordings within one experiment. It also decreases the number of times the turbine has to be run when a parametric study is conducted, again limiting the wear of the turbine.

\section{Software}

Software changes were made to use the full capacity of the segmented mode, decreasing the inter-segment time from $80 \mathrm{~ms}$ to $17 \mu \mathrm{s}$. Furthermore, the number of segments, the number of frames per segment, and the location of the segments can now be chosen arbitrarily.

\section{Fluorescence}

The camera system was adapted to be able to perform fluorescence microscopy on a nanoseconds time scale. To assess the feasibility of a high-speed fluorescence imaging system based on the Brandaris 128, an analysis was made, taking into account all parts that contribute to obtain an acceptable SNR at 25 Mfps: the image sensor, the imaging optics, the fluorescent dye, and the excitation light source. This can be described by the following equation:

$$
\mathrm{SNR} \propto Q E_{\mathrm{CCD}} \times \mathrm{NA} \times \Phi_{\text {photon }},
$$

where $\mathrm{SNR}$ is the signal-to-noise ratio, $Q E_{\mathrm{CCD}}$ is the quantum efficiency of the image sensor, NA is the effective numerical aperture of the optical system, and $\Phi_{\text {photon }}$ is the photon flux generated by the fluorescent object. Improving the image sensor sensitivity would mean replacement of the current Sony ICX-055AL chips; a chip that was chosen specifically for its high sensitivity. The sensitivity per unit area of CCD sensors has not increased much since the introduction of the Brandaris 128. Back-illuminated CCDs have a higher quantum efficiency, but this would increase the sensitivity only by a factor of 2. The use of image intensifiers or electron multiplication applied in EMCCDs will increase the sensitivity by one or perhaps two orders of magnitude, however, these techniques require a large amount of space and the first introduces very high noise levels while the latter requires extensive cooling, which adds to the increased form factor. Further- more, enormous costs would be involved in the replacement of the sensors and controller cards, and the required redesign of the camera interior. The numerical aperture of the microscope system is already optimized by the use of high-NA water immersion objectives. The camera design itself limits the NA through the separation distance between two consecutive channels. At the highest magnification, the width of the lenses that produce the images on the CCDs exactly matches the NA of the beam exiting the microscope, leaving no room for improvement. This implies that the increase in sensitivity must come from the generated fluorescence emission.

The photon flux coming from the sample depends on the excited state kinetics of the fluorescent molecules, assuming a steady state is reached and photobleaching is neglected ${ }^{19}$

$$
\Phi_{\text {photon }}=\frac{\phi}{\tau_{s}} n N_{s}
$$

where $\phi$ is the quantum yield, with a maximum of 1 and many commercially available dyes have an efficiency of approximately $70 \%$ or higher. The fluorescence lifetime $\tau_{s}$ is on the order of nanoseconds. It is an intrinsic property of the fluorescent molecules with only little margin for enhancement. The number of molecules $n$ depends on the concentration of the fluorophore and the surface area available for labeling. Only the fraction of molecules residing in the excited state $N_{s}$ can be exploited for increased fluorescence yield, as the other parameters are largely determined by the type of dye and concentration that can be used, and depends on the object that needs to be labeled. Thus, $N_{s}$ determines the amount of fluorescence emission. $N_{s}$ depends on the rate constant of the excited state $k_{s}\left(k_{s}=\tau_{s}^{-1}\right)$ and the excitation rate $k_{a}$ of the molecules $^{19}$

$$
\begin{gathered}
N_{s}=\frac{k_{a}}{k_{s}+2 k_{a}}, \\
k_{a}=\frac{\sigma \lambda}{h c} I,
\end{gathered}
$$

where $\sigma$ is the absorption cross section of a molecule, $\lambda$ is the excitation wavelength, $h$ is Planck's constant, $c$ is the speed of light, and $I$ is the excitation intensity delivered by the light source. At low excitation intensities $\left(k_{s} \gg k_{a}\right)$, the photon flux is proportional to the intensity of the excitation light. This is the case for a mercury arc burner or high-intensity LED light source

$$
\Phi_{\text {photon }}=\phi \frac{\sigma \lambda}{h c} I n
$$

This suggests that increasing the excitation intensity by several orders of magnitude, for instance, through the use of a laser, should be sufficient to achieve the required improvement to go from a milliseconds exposure time down to a nanoseconds exposure time. However, at high excitation intensities $\left(k_{s} \ll k_{a}\right)$, saturation occurs and the photon flux becomes independent of the excitation intensity

$$
\Phi_{\text {photon }}=\frac{\phi}{2 \tau_{s}} n
$$

This result shows that when the excitation intensity is sufficiently large to cause saturation, the fluorescence signal is 

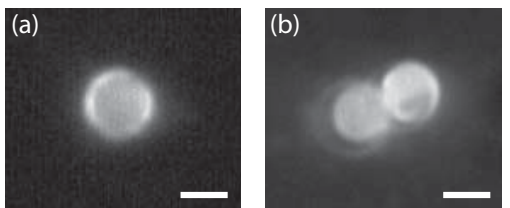

FIG. 4. Fluorescence images recorded with the Brandaris 128 of (a) a fluorescently labeled phospholipid-coated microbubble (500 ns exposure time) and (b) a pair of fluorescently labeled, oil-filled polymeric microcapsules (55 ns exposure time). Scale bars represent $5 \mu \mathrm{m}$. The high pay-load of the polymeric capsules allowed for fluorescence imaging up to the maximum frame rate of the camera.

almost completely determined by the number of dye molecules. For a phospholipid-coated microbubble labeled with a fluorescent dye such as Rhodamine or DiI-C18, the total number of fluorescent molecules is limited by the surface area of the bubble. Nevertheless, preliminary experiments using laser-induced fluorescence (LIF) showed that fluorescence imaging of microbubbles at frame rates exceeding $1 \mathrm{Mfps}$ is feasible. For example, Figure 4 shows a phospholipid-coated microbubble and a pair of oil-filled polymer microcapsules. They were imaged with the Brandaris 128 with an exposure time of 500 and $55 \mathrm{~ns}$, respectively. The high loading capacity of the polymeric capsules allowed for fluorescence imaging up to $25 \mathrm{Mfps}$, which is the maximum frame rate of the camera. The microbubbles, with a much less efficient loading of fluorescent dye molecules, could be imaged up to $2 \mathrm{Mfps}$ in fluorescence.

\section{NEW SYSTEM DESCRIPTION}

\section{A. Dedicated timing controller}

The trigger system consists of a dedicated PC with a PCIbased timer/counter with IO-interfacing (NI 6602, National Instruments, Austin, TX) and a PCI-based programmable delay board (BME sG02p, Bergmann Messgeräte Entwicklung KG, Murnau, Germany). The input for the system is the mirror pulse, produced by an infrared laser/photodiode pair, mounted at $30^{\circ}$ below the optical axis, providing accurate measurement of the instantaneous mirror rotation rate and mirror position. The control software is written in C. It records every mirror pulse interval and saves it for logging purposes. Every $4 \mathrm{~ms}$ the delays of the various trigger signals are adjusted for the running estimate of the mirror speed. Arming of the delay board is combined with an extra control signal such as a manually generated input signal or a preset frame rate.

The accuracy obtained with this system depends on two parts and can be calculated as follows. The first cause of a trigger inaccuracy is the readout of the mirror pulse interval by the timer/counter. By averaging over tens of pulses, the accuracy is increased by decreasing the random timing error from 12.5 ns to a few nanoseconds. The second cause is the change in frame rate, caused by variations in the turbine rotation speed. When the voltage to the MFC is set for a chosen frame rate, the speed will show an overshoot before reaching a stable value. To reduce the wear of the turbine, experiments are now performed around the overshoot, which results in an

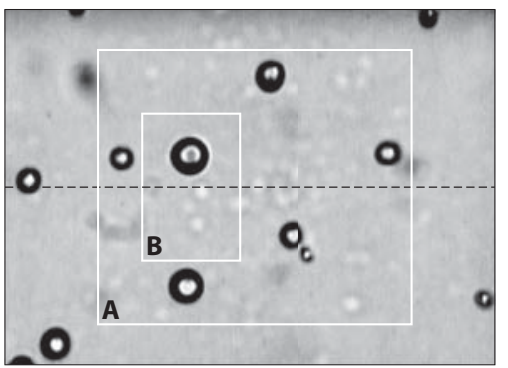

FIG. 5. The region of interest mode lets the user select a region of the full frame to increase the number of full length exposures. The only constraint is that the ROI has to be vertically centered. The marked rectangle "A" has a size of $320 \times 240$ pixels, corresponding to 12 exposures. Rectangle "B" has a size of $100 \times 120$ pixels, corresponding to 72 exposures.

increased variation in the turbine speed during an experiment with a maximum of $15 \%$ per second, resulting in a variation of $0.06 \%$ within one loop of the timing controller. This accuracy also applies to the triggered signals, which, if kept below $50 \mu \mathrm{s}$, results in a timing error of less than $30 \mathrm{~ns}$. These numbers suggest a timing accuracy of less than one single frame at the maximum frame rate and this conclusion has been checked and confirmed by experiments.

\section{B. Region of interest mode}

Instead of using the full frame, a ROI can be selected if there is a need for recording more than 6 exposures using all 128 channels for each exposure. In the ROI mode, the user has the option to select a subsection of the full frame as shown in Figure 5. Because the image size is reduced, the number of images that can be stored per CCD in the onboard RAM is considerably increased.

The horizontal position of the ROI can be selected from 11 pre-defined values and the vertical position is fixed around the center of the frame. The vertical symmetry was chosen because of limited resources of the field-programmable gate array on the CCD-controller. Both the height and width of the ROI can be selected from 9 pre-defined values. The minimum size is $105 \times 78$ pixels and the maximum size is 500 $\times 292$ pixels which is equal to a full frame. Selecting the minimum size of the ROI results in a maximum of 125 exposures, given the $1 \mathrm{MB}$ RAM storage space per channel. In a typical experiment, a ROI of $153 \times 114$ pixels results in 60 exposures within a single experiment. Each exposure requires $80 \mathrm{~ms}$ to be transferred to the onboard memory, leading to a total time of $4.8 \mathrm{~s}$ in which 60 experiments can be recorded.

\section{Segmented mode}

The segmented mode was devised with the purpose to increase the number of recordings within one experiment and to have an inter-segment time in the order of microseconds. The latter requirement was not achieved yet during the introduction of the Brandars 128 because of previous limitations of the timing controller and the limited pulse length and pulse repetition frequency (PRF) of the light source. Adaptations to the software that controls the triggering and to the light source 

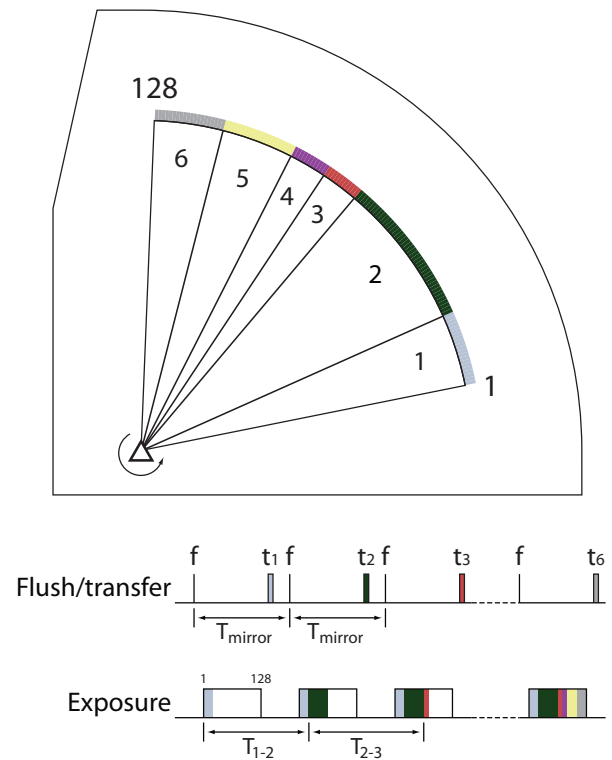

FIG. 6. Schematic representation of the improved segmented mode. The 128 channels can be subdivided into segments with an arbitrary number $(\leq 128)$ of frames. The example shows 6 segments of different length. The timing of the flush and transfer triggers is matched to the mirror period $\left(\mathrm{T}_{\text {mirror }}\right)$. The flush signals (f) are sent to all channels, whereas the transfer signals $\left(\mathrm{t}_{n}\right)$ are segment specific. The time difference between the start of two subsequent segments $\left(\mathrm{T}_{n}-\mathrm{T}_{n+1}\right)$ depends on their position.

have been made to enable subdivision of the 128 channels into arbitrarily chosen segments with a short inter-segment time.

The time between segments can be strongly reduced since the image data are transferred quickly from the sensor into the transport channel before a second exposure, where the data are unaffected by any additional exposures of the CCD. The readout process takes approximately $80 \mathrm{~ms}$, whereas transfer of the image data into the transport channel is completed within $10 \mu \mathrm{s}$. After the recording of the final segment, the image data from all channels are transferred to the RAM, and after $80 \mathrm{~ms}$ the first segment can be exposed again.

The user now has full control over the number of frames in each segment, the position of the frames and the time delay between the segments. Figure 6 shows a timing diagram in which the 6 exposures are depicted horizontally and the different segments within one recording are depicted vertically. The minimum size of a segment is now a single frame and the minimum inter-segment time is equal to the rotation period of the mirror divided by the number of mirror faces. In case of a three-sided mirror rotating at its maximum speed of $20000 \mathrm{rps}$, the inter-segment time is $17 \mu \mathrm{s}$. Because each frame can be allocated separately to a specific segment, segments can contain several frames located randomly within the total of 128 channels.

In order to obtain inter-segment times in the order of the microseconds, the timing sequence and the illumination had to be adjusted. A separate delay generator (Model 555, Berkeley Nucleonics Company) was used to control the triggering of the flush for the CCDs, the ultrasound, and the illumination for each segment. The xenon flash light source used for bright field illumination has a maximum repetition rate of $15 \mathrm{~Hz}$, which is too slow to generate a separate pulse for each segment. On the other hand, a single pulse does not cover all segments, since the discharge pulse length is approximately 40-50 $\mu \mathrm{s}$. Therefore, the electrical discharge circuit of the light source was modified to have a discharge lasting up to $1 \mathrm{~ms}$, thereby allowing all segments to be illuminated by a single flash. The electrical capacitance was increased to maintain sufficient illumination levels, although it was observed that it reduced the lifetime of the light source.

\section{Ultra-high-speed fluorescence imaging setup}

Laser-induced fluorescence is used in many research fields such as the visualization of turbulent mixing flows ${ }^{20}$ or combustion processes ${ }^{21}$ (micro) particle image velocimetry $(\mu \mathrm{PIV}),{ }^{22}$ and molecular spectroscopy. ${ }^{23}$ For measuring velocity fields often dual cavity Nd:YAG lasers provide the fluorescence excitation of micrometer-sized or nanometer-sized tracer particles. The typical PRF of the laser is in the order of $10 \mathrm{~Hz}$, whereas Nd:YLF lasers can achieve a PRF of up to $200 \mathrm{kHz}$. Nevertheless, a separate cavity would be required for each individual frame to be able to do LIF at frame rates exceeding $1 \mathrm{Mfps},{ }^{24}$ rendering pulsed lasers unsuitable especially when taking into account the required power supply and cooling equipment.

Mode-locked lasers have fundamental repetition rates in the order of $100 \mathrm{MHz}$ and higher, fast enough to match the frame rates of the Brandaris 128. The high PRF results in multiple pulses per exposure at a time scale comparable to the fluorescence lifetime of a fluorescent molecule, resulting in a quasi-continuous illumination.

A CW laser does not reach the high peak intensity levels of a pulsed laser, but it can utilize the full exposure time to excite the fluorescent molecules for a prolonged period of time. Moreover, diode-pumped solid-state lasers come in a large variety of wavelengths, they have a small form factor, they do not require extensive cooling, and they are more cost effective than pulsed laser systems. For all these reasons, a 5 W DPSS CW laser (Cohlibri, Lightline, Germany) with a wavelength of $532 \mathrm{~nm}$ was chosen as the fluorescence excitation source.

Its small form factor allowed mounting underneath the camera, as depicted schematically in Figure 7. The modular

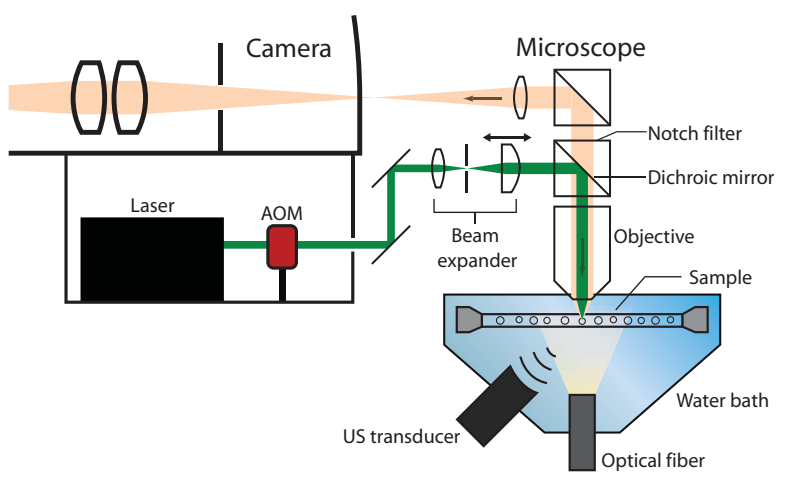

FIG. 7. Schematic view of the experimental used for high-speed fluorescence recordings with the Brandaris 128 . The laser light is focused by the imaging objective onto the sample. The beam-expander filters the laser beam and allows for adjustment of the laser spot size. 
design of the microscope allowed for coupling of the laser light into the optical path of the microscope via a dichroic mirror. Two mirrors align the laser beam onto the beam expander. The beam is passed through a pinhole for spatial filtering, then expanded to a width of $3 \mathrm{~mm}$. By adjusting the position of the plano-convex lens of the beam expander the divergence of the beam can be varied and the spot size at the sample level can be controlled. The dichroic mirror directs the laser light towards the imaging objective that focusses the excitation light onto the sample. A notch filter located above the dichroic mirror filters out any reflected excitation light from the fluorescence emission that has not been filtered out by the dichroic mirror. When recording in bright field with the fluorescence filters in place, $42 \%$ of the xenon illumination is filtered out by the dichroic mirror and notch filter, maintaining sufficient light levels for bright field imaging.

Focusing of the laser light through the imaging objective is necessary to achieve sufficient excitation levels of the fluorescent dye molecules. Consequently, the field of view for fluorescence is limited by the laser spot size. The smallest spot can be achieved by positioning the plano-convex lens such that the beam exiting the beam expander is parallel. A $60 \times$ water immersion objective with a numerical aperture of 0.9 , typically used for microbubble experiments, produces a spot with a full width at half maximum (FWHM) of $1.0 \mu \mathrm{m}$ and a maximum laser intensity of $72 \mathrm{MW} / \mathrm{cm}^{2}$. The spot diameter can be increased by making the laser beam divergent before it enters the objective. This greatly decreases the laser intensity as shown in Figure 8. The optimal spot size depends strongly

(a)
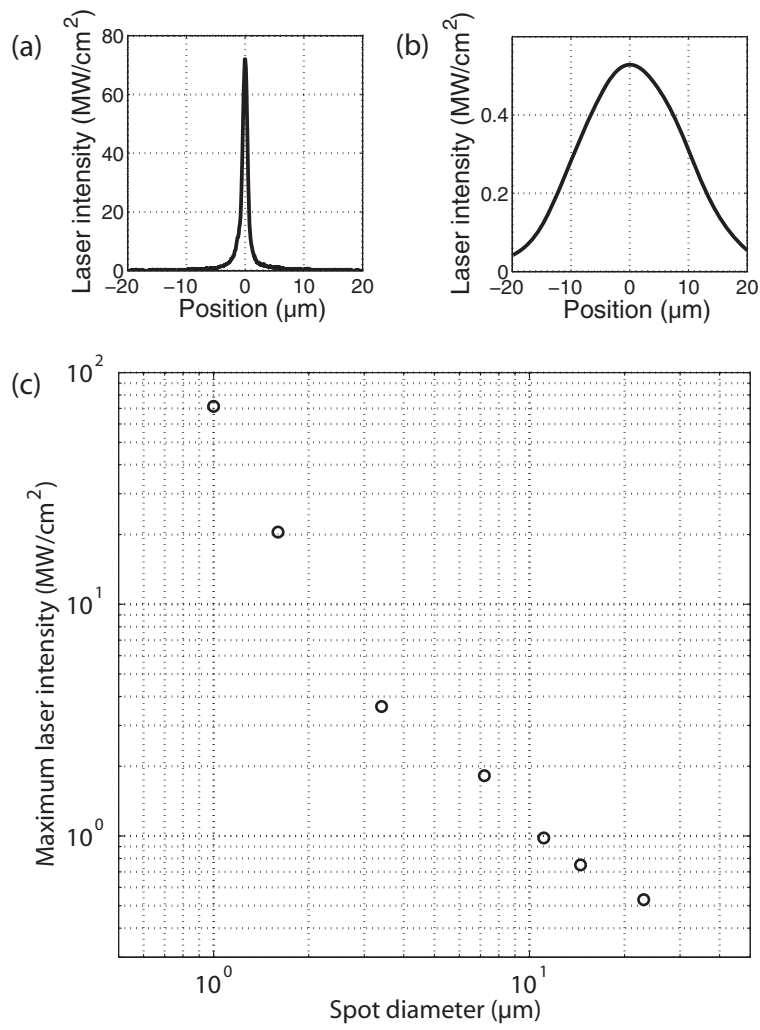

FIG. 8. (a) Laser beam profile for a spot with FWHM $1.0 \mu \mathrm{m}$ and (b) $23 \mu \mathrm{m}$. (c) The measured maximum excitation intensity as a function of laser spot size for a $60 \times$ objective with a NA of 0.9 . on the observed sample and should be matched to the desired field of view.

Direct modulation of the voltage supplied to the laser head allows for a maximum switching rate of $1 \mathrm{kHz}$, which results in a rise time longer than the recording time of the Brandaris 128. The amount of photobleaching generated during the startup of the laser can be significant and at a millisecond time scale, unwanted thermal effects can play a role. Heating of the sample should be avoided especially when experiments are performed on biological samples. To facilitate a faster rise time and shorter pulses, the laser was gated by an acousto-optic modulator (AOM) (AOTF.nC-VIS, AA optoelectronic, France) positioned in between the laser and the beam expander. The laser beam was sent through the AOM unfocussed to minimize power loss, although this resulted in a slower switching speed. The light transmitting efficiency of the AOM is $92 \%$, resulting in a maximum power of $4.8 \mathrm{~W}$ for gate times of $5 \mu$ s and longer, corresponding to a rise and fall time of $5 \mu \mathrm{s}$ as shown in Figure 9. Shorter gate times result in decreased laser power.

With the fluorescence module in place, the Brandaris 128 configuration gives rise to three illumination options: bright field, fluorescence, and the combination of both illumination techniques. A digital delay generator (Model 575, Berkeley Nucleonics Corporation, San Rafael, CA) is used to trigger
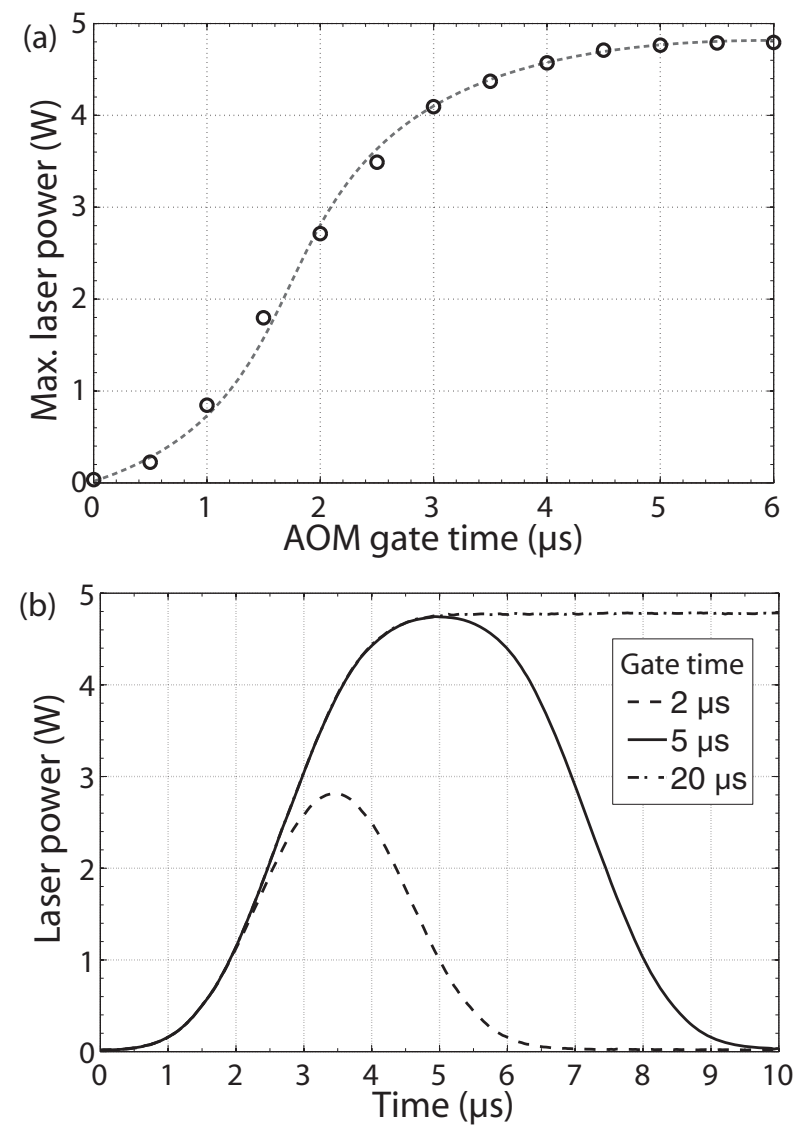

FIG. 9. (a) Maximum laser power for different gate times of the AOM. A maximum power of $4.8 \mathrm{~W}$ was obtained for gate times of $5 \mu$ s and longer. (b) Pulse shape for three different gate times of the AOM. The rise and fall time of the AOM is $5 \mu \mathrm{s}$. 


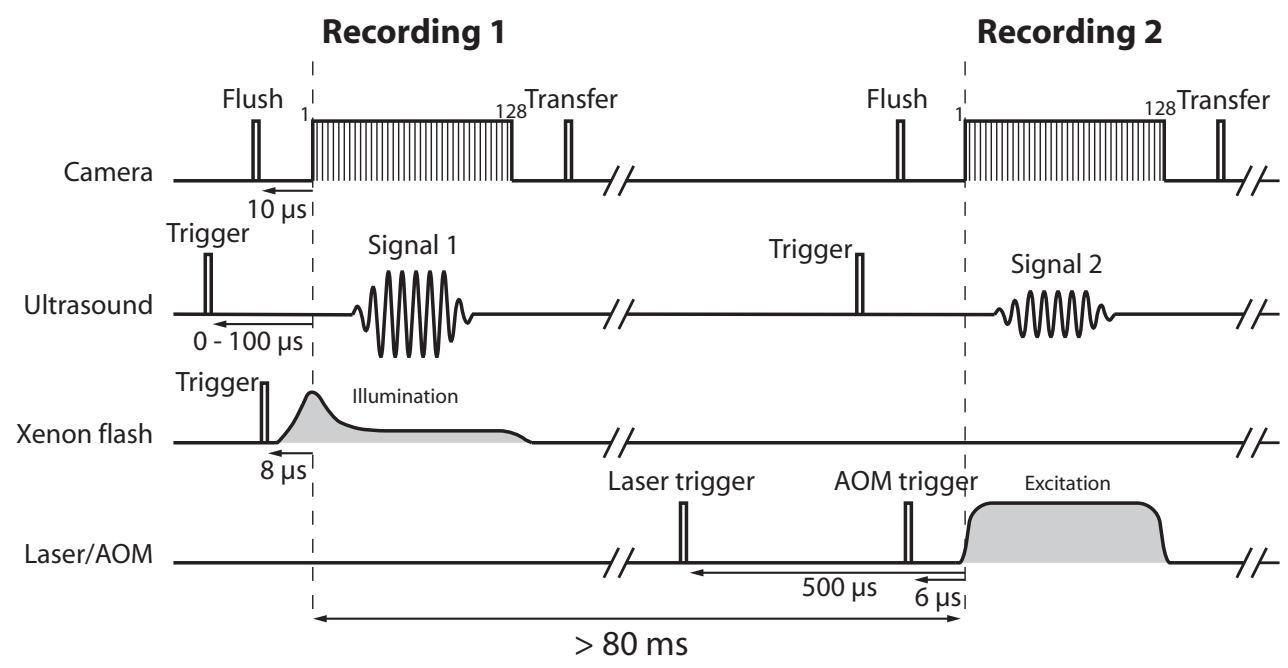

FIG. 10. Timing diagram for two subsequent recordings within a typical experiment. Before each exposure the CCDs are flushed and after capturing frame 128 the image data are transferred to the internal RAM. The xenon flash is triggered several microseconds before exposure 1 to start a bright field recording and before exposure 2 the laser and the AOM are triggered to initiate the fluorescence recording. Separately pre-programmed ultrasound signals are sent for each exposure.

the laser and the AOM, and the xenon strobe, giving full control over the illumination methods. For each individual recording within one experiment, the illumination method can be predefined. In the case of 6 full-frame recordings, for instance, the first 3 can be captured in bright field and the following 3 in fluorescence. Other options are alternating bright field and fluorescence or even 12 combined recordings of which each recording is split into two virtual segments: the first 64 frames are recorded in bright field and the second 64 frames are recorded in fluorescence.

Experiments performed with the Brandaris 128 typically involve ultrasonic excitation of microbubbles or droplets. For this purpose a separate, pre-programmed ultrasound signal is uploaded for each recording to an arbitrary waveform generator (Model 8026, Tabor Electronics Ltd, Israel). A negative time delay is set in the timing controller to take into account the travel time of the ultrasound. Figure 10 shows the timing diagram for two consecutive recordings within one experiment, the first being a bright field recording and the second being a fluorescence recording.

\section{RESULTS}

\section{A. Microbubble spectroscopy using ROI mode}

In previous research, the segmented mode was used to perform a large set of experiments in a single run of the Brandaris 128. Van der Meer et al. subjected a single microbubble to a scan of 24 different frequencies to construct a resonance curve by using 4 segments of 32 frames whereas Emmer et al. ${ }^{10}$ used 12 segments of 64 frames to vary the pressure for a constant frequency. Overvelde et al. ${ }^{25}$ operated the Brandaris 128 in the normal mode to obtain the behavior of a single microbubble for 8 different pressures and 12 different frequencies. In order to use all 128 frames for each exposure, it required in total 16 experiments which takes in total approximately half an hour to record.
By using the new ROI mode (Sec. II B), Faez et al. ${ }^{26}$ were able to record 49 exposures of a single microbubble within a single run of the camera, with the purpose to study its subharmonic behavior. In the first exposure, no ultrasound was applied to determine the resting radius of the bubble. In the subsequent 48 exposures, the frequency was swept in 16 steps from 4 to $7 \mathrm{MHz}$ at an acoustic pressure of $50 \mathrm{kPa}$, followed by 16 frequency steps at $100 \mathrm{kPa}$ and finally 16 frequency steps at $120 \mathrm{kPa}$. Thus, within $5 \mathrm{~s} 3$ resonance curves of a single microbubble were measured. From each exposure the diameter of the bubble as a function of time was determined. From this the maximum amplitude of oscillation at the subharmonic frequency was extracted and plotted against frequency as shown in Figure 11.

\section{B. Imaging at multiple time scales using segmented mode}

A typical recording of the Brandaris 128 at $10 \mathrm{Mfps}$ has a duration of $13 \mu \mathrm{s}$. This is very short as compared to the time

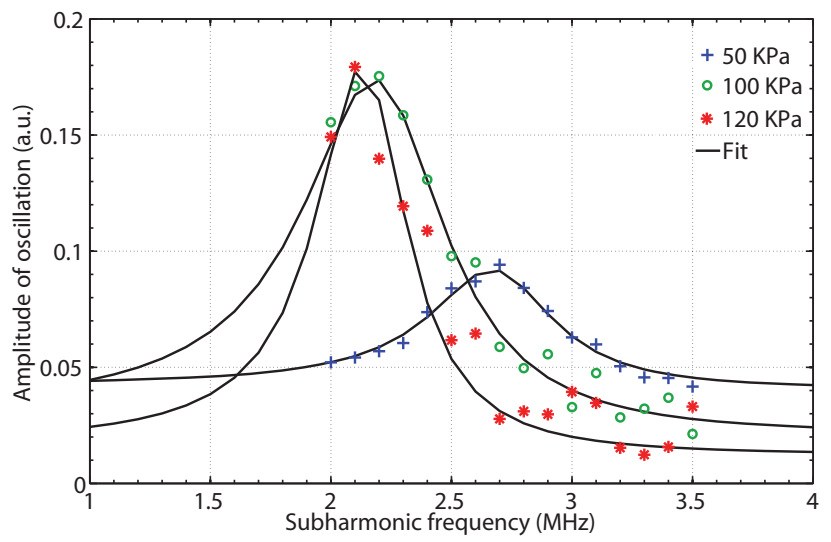

FIG. 11. Three resonance curves of the subharmonic response of a single microbubble. The set of curves was measured within $5 \mathrm{~s}$ in a single run of the camera. 


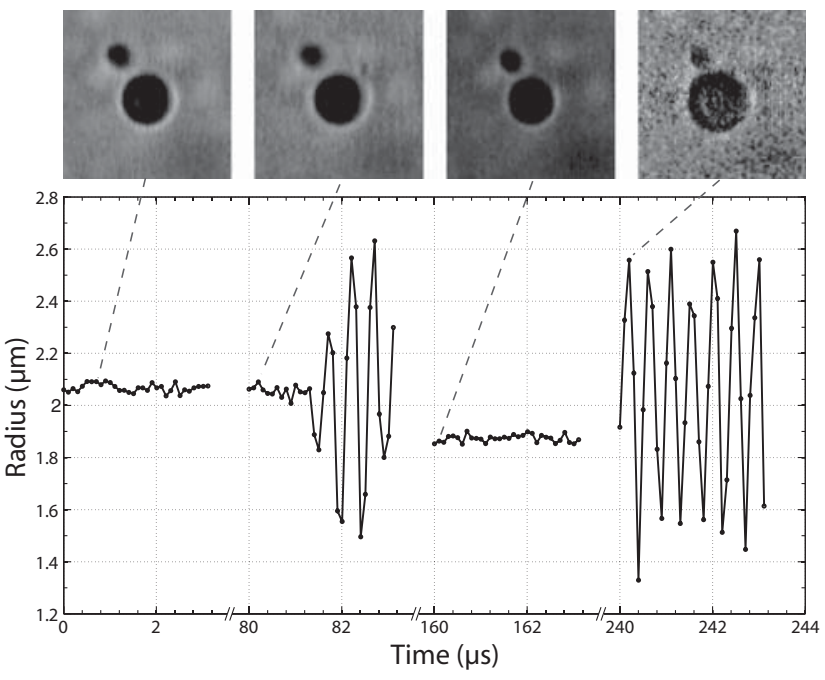

FIG. 12. The radial excursion of a microbubble in four segments with an inter-segment time of $80 \mu \mathrm{s}$. The bubble was insonified at $2.25 \mathrm{MHz}$ in segments 2 and 4 . The difference in resting radius between segments 2 and 3 indicates bubble shrinkage on a microseconds time scale.

between recordings, which is $80 \mathrm{~ms}$ or longer. Two consecutive recordings can therefore be considered to be completely separate experiments, as the objects in the field of view have sufficient time to return to an equilibrium state after ultrasonic excitation. By decreasing the inter-segment time down to the order of microseconds, transient effects can be studied in more detail, while maintaining the high time resolution within the recording itself. It is known, for instance, that lipid shedding of phospholipid-coated microbubbles due to large amplitude oscillations causes shrinkage of a bubble over time. ${ }^{27}$ The improved segmented mode allows us to study this effect at much shorter time scales.

As an example of the improved segmented mode, the frame rate was set to $10 \mathrm{Mfps}$, resulting in a mirror period of $120 \mu \mathrm{s}$. The mirror mounted during the experiment had three reflective sides, which allowed for an inter-segment time of
$40 \mu$ s or any multiple of that number. In this case, the intersegment time was set to $80 \mu \mathrm{s}$.

The 128 CCDs were divided into four segments of 32 frames. A phospholipid-coated microbubble was insonified at $2.25 \mathrm{MHz}$ in segments 2 and 4 . Figure 12 shows the radial excursion of the microbubble in the four segments, where oscillations are visible in the second and fourth segment. The decrease in the resting radius of the bubble between segments 2 and 3 shows that bubble shrinkage can be significant on a time scale of $80 \mu \mathrm{s}$.

\section{Ultra-high-speed fluorescence imaging of microbubbles}

Phospholipid-coated microbubbles, to be used as ultrasound contrast agents, were produced in-house following the method of Klibanov et al. ${ }^{28}$ In the final production step, the microbubbles were washed with the fluorescent dye DiI. The final concentration of dye molecules in the lipid shell was low, considering that DiI is located in between the distearoyl phosphatidylethanolamine polyethylene glycol (DSPE-PEG) molecules that make up only $5 \%$ of the total shell material.

The microbubbles were put into an acoustically and optically transparent OptiCell ${ }^{\mathrm{TM}}$ container and insonified at a frequency of $300 \mathrm{kHz}$ with a pressure amplitude of $150 \mathrm{kPa}$ for a period of 10 cycles. A single microbubble was visualized using a $60 \times$ water-immersion objective. The microbubble oscillations were recorded alternatingly in bright field (exposures 1,3 , and 5) and in fluorescence (exposures 2, 4, and 6) at a frame rate of $1.5 \mathrm{Mfps}$.

Figures 13(a) and 13(b) show still frames from the bright field and the consecutive fluorescence exposure. The radial intensity profiles, depicted in Figure 13(c), show the complex ring structure of the bright field image, due to diffraction and Mie scattering from the microbubble of the bright field illumination. The fluorescence intensity profile shows a simpler profile of the microbubble at a higher contrast; the contrast ratio between the center of the bubble and the background is 1.5
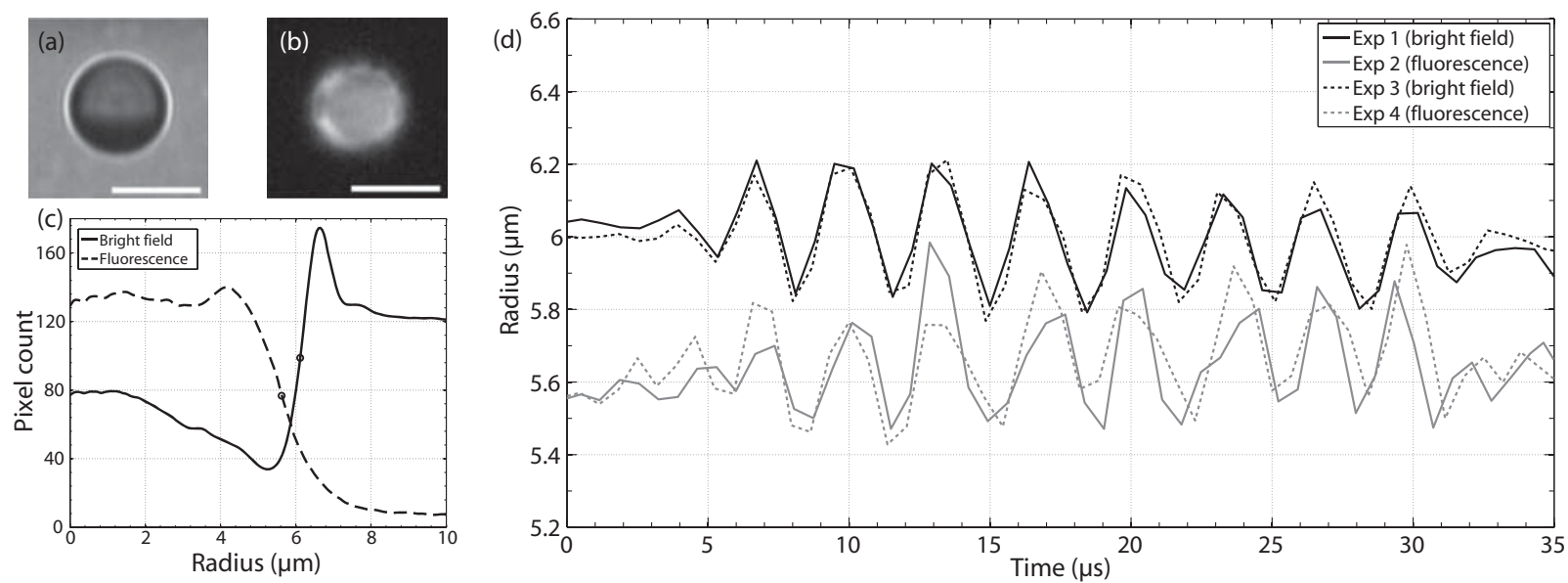

FIG. 13. (a) Still frame from the bright field exposure and (b) from the fluorescence recording of an ultrasound contrast agent microbubble. The scale bar indicates $6 \mu \mathrm{m}$. (c) Radial intensity profiles for bright field imaging (black) and fluorescence imaging (gray). The circles indicate the maximum of the first derivative of the profile, where the radius is determined by the analysis. (d) Radius time curves of the microbubble oscillations. The bright field recordings (black) show a larger radius than the fluorescence recordings (gray). 
for the bright field image and 13 for the fluorescence image. Furthermore, while the bright field images miss all information of the nanometer scale coating material, the fluorescence image shows the inhomogeneities in the distribution of dye molecules over the bubble shell.

The microbubble oscillations were analyzed using MATLAB. The contour of the bubble was tracked in each frame by finding the maximum and minimum slope, respectively, for all angular positions on the bubble rim. The resulting radius-time curve is plotted in Figure 13(d). The bright field traces show great similarity, as well as the fluorescence traces. Both imaging methods capture the dynamics of the bubble oscillations, however, there is a systematically lower radius obtained for the fluorescence image. This can also be seen in Figure 13(c). The minimum slope of the fluorescence image is located at a smaller radius than the maximum slope of the bright field profile. This example demonstrates the importance of fluorescence imaging at these length scales, where diffraction and Mie scattering dominate the bright field image of the bubble with a size in the order of the wavelength of the light. Besides the optical imaging system, i.e., the objective lens, mirror, relay lenses, etc., the illumination system also influences the final image. As the fluorescence emission originates from the object itself, the final image does not suffer from a complex scattering and diffraction pattern.

In order to generate sufficient fluorescence signal the excitation irradiance levels have to be substantial, causing photobleaching of the excited dye molecules, even at the microseconds time scale. Figure 14 shows the relative mean fluorescence intensity of the microbubble depicted in Figure 13 over the course of a single exposure. Within $5 \mu \mathrm{s}$, the intensity has dropped to half of its original value, resulting in a significant loss of signal-to-noise ratio. Experiments with bubbles that were not excited by ultrasound (and as a consequence had a constant radius) showed that the photobleaching does not influence the measured radius. The second curve shows that the photobleaching is largely reversible, indicated by a maximum intensity of the second exposure of $84 \%$ as compared to the first one.

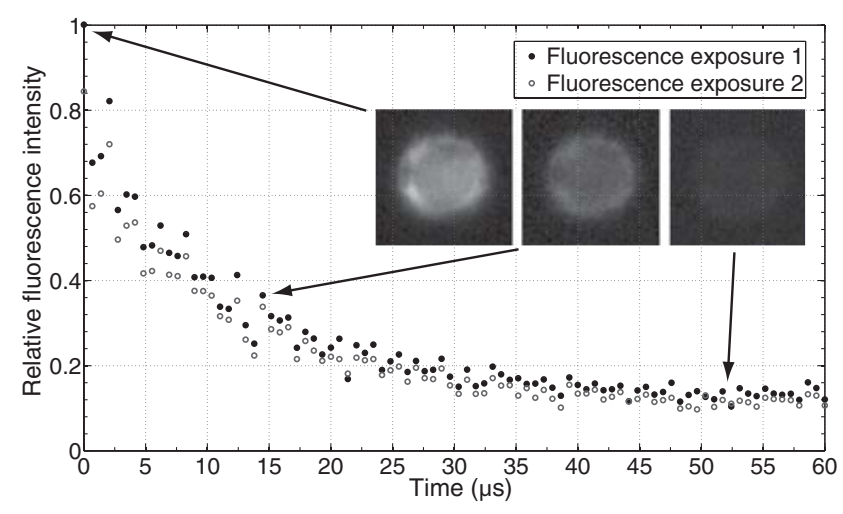

FIG. 14. Fluorescence intensity of a fluorescently labeled microbubble as a function of time. The solid circles show the relative fluorescence intensity over the period of the first exposure to continuous laser excitation light. In the second exposure (open circles), the fluorescence partially recovers to $84 \%$ of the initial value.

\section{DISCUSSION}

The region of interest mode has greatly enhanced the capabilities of the Brandaris 128, by allowing a single experiment to capture the behavior of a microbubble for a large parameter set. It drastically reduces the record time and limits the wear of the turbine and saves on the usage of helium. Furthermore, a microbubble can be studied extensively in a short period of time, reducing the influence of experimental parameters such as temperature, shell composition, local gas concentration, etc. However, the increased number of recordings per experiment causes a somewhat longer download time to transfer the image data to the PC, as the ROI images are exported at full-frame image size by adding a black background around the ROI. Nevertheless, a significant time gain is achieved.

Using the segmented mode, multiple time scales can now be captured within a single recording in order to study phenomena with high temporal resolution over a prolonged period of time. The challenge in this operating mode is the bright field illumination, since only a single discharge from a xenon flash light source can be used and high input energy levels are required that lead to increased deterioration of the lifetime of the light source. Employing multiple light sources coupled into a single optical fiber can be used to overcome this problem, although this would still limit the number of segments. Continuous light sources are not an option, as they do not reach the required light intensity, and laser bright field illumination leads to severe loss in image quality due to speckles and interference patterns, caused by spatial and temporal coherence of the light. This issue can be resolved by using laserinduced fluorescence for back illumination, as described by Van der Bos et al. ${ }^{18}$ However, a pulsed laser is required to obtain sufficient excitation levels, complicating the practical implementation of this illumination technique for time-resolved imaging.

To our knowledge this is the first time fluorescence recordings of an oscillating microbubble at a frame rate exceeding $1 \mathrm{Mfps}$ and a submicrometer resolution have been performed. This demonstrates that the sensitivity of the Brandaris 128 imaging facility has been increased tremendously. This opens up an exciting new field of research applications for the camera, allowing for the visualization of phenomena such as ultrasound-triggered drug release and uptake and flow visualization using nanoseconds time-resolved $\mu \mathrm{PIV}$ and PTV. Since no transmitted light is required for fluorescence imaging and the bubble-background contrast is greatly enhanced, optical in vivo studies of ultrasound contrast agents can be performed. In all experiments, but especially when biological samples are used, thermal effects of the laser illumination due to unwanted absorption have to be considered. When long pulses are used for imaging at a lower frame rate or when the concentration of dye is very high, care should be taken to avoid heating, and which may potentially lead to vaporization. Furthermore, the amount of photobleaching has to be minimized, to ensure a sufficient signal-to-noise ratio. Since the photobleaching depends on many parameters, such as the pulse length of the laser, the laser intensity, its spot size, but also the dye concentration, an assessment should be made 
for each individual experiment to find a satisfactory compromise between fluorescence yield and photobleaching.

With all these new features in place, one issue remains unresolved, namely, the maximum frame rate of the camera. Redesigning the optical configuration by placing the image arc further away from the mirror to fit more channels in is an option, although the costs would be very high and it would result in a reduced numerical aperture. Driving the turbine at higher speeds requires minimizing the viscous drag by running it on hydrogen instead of helium or by running it in a vacuum. Even if this can be achieved, the stability of the turbine system can then become the limiting factor. When frame rates exceeding $20 \mathrm{Mfps}^{26}$ are used, care must be taken to avoid excessive heat buildup and wear of the bearings of the mirror turbine. An increase in speed of a factor of two can theoretically be achieved by splitting the beam coming from the microscope and thereby making two separate images per CCD-sensor. However, the NA of the camera would become a factor 2 lower in addition to the reduced exposure time, resulting in a real challenge to collect sufficient photons per image.

\section{CONCLUSIONS}

Since its introduction ten years ago, the Brandaris 128 has been a unique ultra-high-speed imaging system. The improvements that were made in the last decade further contribute to the versatility of the system. By increasing the number of frames from 768 to 16000 , a single run of the camera can now record up to 125 unique recordings within several seconds. The time between separate recordings has been reduced by three orders of magnitude from $80 \mathrm{~ms}$ to $17 \mu \mathrm{s}$, enabling imaging at multiple time scales ranging from nanoseconds to milliseconds. The incorporated fluorescence module unites all the advantages of fluorescence imaging such as enhanced contrast and resolution to time-resolved high-speed imaging. Combined with the maximum frame rate of $25 \mathrm{Mfps}$, the Brandaris 128 remains an exceptional camera with great potential for future research.

The Brandaris camera has been focused on studies related to ultrasound contrast agents. Past applications with the camera also include inkjet printing, pulmonary drug delivery spray formation, semiconductor cleaning, hydrodynamic and acoustic cavitation root canal endodontic therapy, lab-on-a-chip micro- and nanofluidics, and particle tracking and particle imaging velocimetry. Future applications of the camera are foreseen in plasma and lightning sparks, laser materials processing, protein folding dynamics, and turbulent combustion.

\section{ACKNOWLEDGMENTS}

The authors would like to thank Geert Springeling, Robert Beurskens, Gert-Wim Bruggert, and Martin Bos for their technical contributions, and Frédéric Lemmel for re- designing the CCD controller software. Klazina Kooiman is also gratefully acknowledged for preparing the fluorescently labeled microbubbles.

${ }^{1}$ C. T. Chin, C. Lancée, J. Borsboom, F. Mastik, M. E. Frijlink, N. de Jong, M. Versluis, and D. Lohse, Rev. Sci. Instrum. 74(12), 5026 (2003).

${ }^{2}$ J. Sijl, M. L. J. Overvelde, B. Dollet, V. Garbin, N. de Jong, D. Lohse, and M. Versluis, J. Acoust. Soc. Am. 129(4), 1729 (2011).

${ }^{3}$ W. van Hoeve, S. Gekle, J. H. Snoeijer, M. Versluis, M. P. Brenner, and D. Lohse, Phys. Fluids 22(12), 122003 (2010).

${ }^{4}$ A. G. Zijlstra, Ph.D. dissertation, University of Twente, Enschede, The Netherlands, 2011; see http://doc.utwente.nl/77876/1/ thesis_A_Zijlstra.pdf.

${ }^{5}$ V. Parker and C. Roberts, High Speed Photography and Photonics, edited by S. F. Ray (SPIE, Bellingham, WA, 1997).

${ }^{6}$ E. A. Igel and M. Kristiansen, Rotating-Mirror Streak and Framing Cameras (SPIE, Bellingham, WA, 1997), Vol. PM43.

${ }^{7}$ S. M. van der Meer, B. Dollet, M. M. Voormolen, C. T. Chin, A. Bouakaz, N. de Jong, M. Versluis, and D. Lohse, J. Acoust. Soc. Am. 121(1), 648 (2007).

${ }^{8}$ P. Marmottant, S. M. van der Meer, M. Emmer, M. Versluis, N. de Jong, S. Hilgenfeldt, and D. Lohse, J. Acoust. Soc. Am. 118(6), 3499 (2005).

${ }^{9}$ N. de Jong, M. Emmer, C. T. Chin, A. Bouakaz, F. Mastik, D. Lohse, and M. Versluis, Ultrasound Med. Biol. 33(4), 653 (2007).

${ }^{10}$ M. Emmer, A. van Wamel, D. E. Goertz, and N. de Jong, Ultrasound Med. Biol. 33(6), 941 (2007).

${ }^{11}$ B. Dollet, S. M. van der Meer, V. Garbin, N. de Jong, D. Lohse, and M. Versluis, Ultrasound Med. Biol. 34(9), 1465 (2008).

${ }^{12}$ J. Sijl, B. Dollet, M. Overvelde, V. Garbin, T. Rozendal, N. de Jong, D. Lohse, and M. Versluis, J. Acoust. Soc. Am. 128(5), 3239 (2010).

${ }^{13}$ V. Garbin, D. Cojoc, E. Ferrari, E. Di Fabrizio, M. L. J. Overvelde, S. M. van der Meer, N. de Jong, D. Lohse, and M. Versluis, Appl. Phys. Lett. 90, 114103 (2007)

${ }^{14}$ K. Kooiman, M. R. Böhmer, M. Emmer, H. J. Vos, C. Chlon, W. T. Shi, C. S. Hall, S. de Winter, K. Schroen, M. Versluis, N. de Jong, and A. van Wamel, J. Controlled Release 133(2), 109 (2009).

${ }^{15}$ A. van Wamel, K. Kooiman, M. Harteveld, M. Emmer, F. J. ten Cate, M. Versluis, and N. de Jong, J. Controlled Release 112(2), 149 (2006).

${ }^{16}$ A. van der Bos, Ph.D. dissertation, University of Twente, Enschede, The Netherlands, 2011; see http://doc.utwente.nl/75595/1/thesis_A_van_ den_Bos.pdf.

${ }^{17}$ A. G. Zijlstra, T. Janssens, K. Wostyn, M. Versluis, P. W. Mertens, and D. Lohse, Solid State Phenom. 145(7), 7 (2009).

${ }^{18}$ A. van der Bos, A. Zijlstra, E. Gelderblom, and M. Versluis, Exp. Fluids 51(5), 1283 (2011).

${ }^{19} \mathrm{R}$. Loudon, The Quantum Theory of Light, 3rd ed. (Oxford University Press, New York, 2001).

${ }^{20}$ J. P. Crimaldi, Exp. Fluids 44(6), 851 (2008).

${ }^{21}$ A. Leipertz, A. Braeuer, J. Kiefer, A. Dreizler, and C. Heeger, Handbook of Combustion (Wiley-VCH, Verlag, 2010), p. 219.

${ }^{22}$ S. T. Wereley and C. D. Meinhart, Ann. Rev. Fluid Mech. 42(1), 557 (2010).

${ }^{23}$ F. C. De Lucia, R. S. Harmon, K. L. McNesby, R. J. Winkel, and A. W. Miziolek, Appl. Opt. 42(30), 6148 (2003).

${ }^{24}$ T. Ding, Th. H. van der Meer, M. Versluis, M. Golombok, J. Hult, M. Alden, and C. F. Kaminski, in Proceedings of the Third International Symposium on Turbulence, Heat and Mass Transfer, edited by Y. Nagano, K. Hanjalic, and T. Tsuji (Aichi Shuppan, 2000), p. 857.

${ }^{25}$ M. L. J. Overvelde, V. Garbin, J. Sijl, B. Dollet, N. de Jong, D. Lohse, and M. Versluis, Ultrasound Med. Biol. 36(12), 2080 (2010).

${ }^{26}$ T. Faez, M. Emmer, M. Docter, J. Sijl, M. Versluis, and N. de Jong, Ultrasound Med. Biol. 37(6), 958 (2011).

${ }^{27}$ F. Guidi, H. J. Vos, R. Mori, N. de Jong, and P. Tortoli, IEEE Trans. Ultrason. Ferroelectr. Freq. Control 57(1), 193 (2010).

${ }^{28}$ A. L. Klibanov, P. T. Rasche, M. S. Hughes, J. K. Wojdyla, K. P. Galen, J. H. Wible, and G. H. Brandenburger, Invest. Radiol. 39(3), 187 (2004). 